\title{
Morbo Serpentino
}

The approach to clinical conundrums by an expert clinician is revealed through the presentation of an actual patient's case in an approach typical of a morning report. Similarly to patient care, sequential pieces of information are provided to the clinician, who is unfamiliar with the case. The focus is on the thought processes of both the clinical team caring for the patient and the discussant.

This icon represents the patient's case. Each paragraph that follows represents the discussant's thoughts.

Helene Møller Nielsen, MD*, Shakil Shakar, MD¹, Ulla Weinreich MD¹, Mary Hansen MD², Rune Fisker, $\mathrm{MD}^{3}$, Thomas E. Baudendistel, MD', Paul Aronowitz MD ${ }^{5}$

${ }^{1}$ Department of Pulmonary Medicine, Aalborg University Hospital, Aalborg, Denmark; '2Department of Pathology, Aalborg University Hospital, Aalborg, Denmark; ${ }^{3}$ Department of Nuclear Medicine, Aalborg University Hospital, Aalborg, Denmark; ${ }^{4}$ Department of Medicine, Kaiser Permanente, Oakland, California; ${ }^{5}$ Department of Medicine, University of California, Davis, California.

A 58-year-old Danish man presented to an urgent care center due to several months of gradually worsening fatigue, weight loss, abdominal pain, and changes in vision. His abdominal pain was diffuse, constant, and moderate in severity. There was no association with meals, and he reported no nausea, vomiting, or change in bowel movements. He also said his vision in both eyes was blurry, but denied diplopia and said the blurring did not improve when either eye was closed. He denied dysphagia, headache, focal weakness, or sensitivity to bright lights.

Fatigue and weight loss in a middle-aged man are nonspecific complaints that mainly help to alert the clinician that there may be a serious, systemic process lurking. Constant abdominal pain without nausea, vomiting, or change in bowel movements makes intestinal obstruction or a motility disorder less likely. Given that the pain is diffuse, it raises the possibility of an intraperitoneal process or a process within an organ that is irritating the peritoneum.

Worsening of vision can result from disorders anywhere along the visual pathway, including the cornea (keratitis or corneal edema from glaucoma), anterior chamber (uveitis or hyphema), lens (cataracts, dislocations, hyperglycemia), vitreous humor (uveitis), retina (infections, ischemia, detachment, diabetic retinopathy), macula (degenerative disease), optic nerve (optic neuritis), optic chiasm, and the visual projections through the hemispheres to the occipital lobes. To narrow the differential diagnosis, it would be important to inquire about prior eye problems, to measure visual acuity and intraocular pressure, to perform fundoscopic and slitlamp exams to detect retinal and anterior chamber disorders,

\footnotetext{
*Address for correspondence and reprint requests: Helene Møller Nielson, MD, Department of Pulmonary Medicine, Aalborg University Hospital, Denmark; Telephone: 459-766-4748; Fax: 459-762-6407; E-mail: hemoni@rn.dk.

Received: October 21, 2016; Revised: March 21, 2017;

Accepted: March 24, 2017
}

2017 Society of Hospital Medicine DOI 10.12788/jhm.2821 respectively, and to assess visual fields. An afferent pupillary defect would suggest optic nerve pathology.

Disorders that could unify the constitutional, abdominal, and visual symptoms include systemic inflammatory diseases, such as sarcoidosis (which has an increased incidence among Northern Europeans), tuberculosis, or cancer. While diabetes mellitus could explain his visual problems, weight loss, and fatigue, the absence of polyuria, polydipsia, or polyphagia argues against this possibility.

The patient had hypercholesterolemia and type 2 diabetes mellitus. Medications were metformin, atorvastatin, and glimepiride. He was a former smoker with 23 pack-years and had quit over 5 years prior. He had not traveled outside of Denmark in 2 years and had no pets at home. He reported being monogamous with his same-sex partner for the past 25 years. He had no significant family history, and he worked at a local hospital as a nurse. He denied any previous ocular history.

On examination, the pulse was 67 beats per minute, temperature was 36.7 degrees Celsius, respiratory rate was 16 breaths per minute, oxygen saturation was $99 \%$ while breathing ambient air, and blood pressure was $132 / 78$. Oropharynx demonstrated no thrush or other lesions. The heart rhythm was regular and there were no murmurs. Lungs were clear to auscultation bilaterally. Abdominal exam was normal except for mild tenderness upon palpation in all quadrants, but no masses, organomegaly, rigidity, or rebound tenderness were present. Skin examination revealed several subcutaneous nodules measuring up to $0.5 \mathrm{~cm}$ in diameter overlying the right and left posterolateral chest walls. The nodules were rubbery, pink, nontender, and not warm nor fluctuant. Visual acuity was reduced in both eyes. Extraocular movements were intact, and the pupils reacted to light and accommodated appropriately. The sclerae were injected bilaterally. The remainder of the cranial nerves and neurologic exam were normal. Due to the vision loss, the patient was referred to an ophthalmologist who diagnosed bilateral anterior uveitis. 
Though monogamous with his male partner for many years, it is mandatory to consider complications of human immunodeficiency virus infection (HIV). The absence of oral lesions indicative of a low CD4 count, such as oral hairy leukoplakia or thrush, does not rule out HIV disease. Additional history about his work as a nurse might shed light on his risk of infection, such as airborne exposure to tuberculosis or acquisition of blood-borne pathogens through a needle stick injury. His unremarkable vital signs support the chronicity of his medical condition.

Uveitis can result from numerous causes. When confined to the eye, uncommon hereditary and acquired causes are less likely. In many patients, uveitis arises in the setting of systemic infection or inflammation. The numerous infectious causes of uveitis include syphilis, tuberculosis, toxoplasmosis, cat scratch disease, and viruses such as HIV, West Nile, and Ebola. Among the inflammatory diseases that can cause uveitis are sarcoidosis, inflammatory bowel disease, systemic lupus erythematosus, Behçet disease, and Sjogren syndrome.

Several of these conditions, including tuberculosis and syphilis, may also cause subcutaneous nodules. Both tuberculosis and syphilis can cause skin and gastrointestinal disease. Sarcoidosis could involve the skin, peritoneum, and uvea, and is a possibility in this patient. The dermatologic conditions associated with sarcoidosis are protean and include granulomatous inflammation and nongranulomatous processes such as erythema nodosum. Usually the nodules of erythema nodosum are tender, red or purple, and located on the lower extremities. The lack of tenderness points away from erythema nodosum in this patient. Metastatic cancer can disseminate to the subcutaneous tissue, and the patient's smoking history and age mandate we consider malignancy. However, skin metastases tend to be hard, not rubbery.

A cost-effective evaluation at this point would include syphilis serologies, HIV testing, testing for tuberculosis with either a purified protein derivative test or interferon gamma release assay, chest radiography, and biopsy of 1 of the lesions on his back.

Laboratory data showed 12,400 white blood cells per cubic milliliter (64\% neutrophils, $24 \%$ lymphocytes, $9 \%$ monocytes, $2 \%$ eosinophils, $1 \%$ basophils), hemoglobin $7.9 \mathrm{~g} / \mathrm{dL}$, mean corpuscular volume $85 \mathrm{fL}$, platelets 476,000 per cubic milliliter, C-reactive protein $43 \mathrm{mg} / \mathrm{dL}$ (normal $<8 \mathrm{mg} / \mathrm{L}$ ), gamma-glutamyl-transferase $554 \mathrm{IU} / \mathrm{L}$ (normal range 0-45), alkaline phosphatase $865 \mathrm{U} / \mathrm{L}$ (normal range 60-200), and erythrocyte sedimentation rate (ESR) $71 \mathrm{~mm}$ per hour. International normalized ratio was 1.0 , albumin was $3.0 \mathrm{mg} / \mathrm{dL}$, activated partial thromboplastin time was 32 seconds (normal 22 to 35 seconds), and bilirubin was $0.3 \mathrm{mg} / \mathrm{dL}$. Antibodies to HIV, hepatitis $\mathrm{C}$, and hepatitis $\mathrm{B}$ surface antigen were not detectable. Electrocardiography (ECG) was normal. Plain radiograph of the chest demonstrated multiple nodular lesions bilaterally measuring up to $1 \mathrm{~cm}$ with no cavitation. There was a left pleural effusion.
The history and exam findings indicate a serious inflammatory condition affecting his lungs, pleura, eyes, skin, liver, and possibly his peritoneum. In this context, the elevated C-reactive protein and ESR are not helpful in differentiating inflammatory from infectious causes. The constellation of uveitis, pulmonary and cutaneous nodules, and marked abnormalities of liver tests in a middle-aged man of Northern European origin points us toward sarcoidosis. Pleural effusions are not common with sarcoidosis but may occur. However, to avoid premature closure, it is important to consider other possibilities.

Metastatic cancer, including lymphoma, could cause pulmonary and cutaneous nodules and liver involvement, but the chronic time course and uveitis are not consistent with malignancy. Tuberculosis is still a consideration, though one would have expected him to report fevers, night sweats, and, perhaps, exposure to patients with pulmonary tuberculosis in his job as a nurse. Multiple solid pulmonary nodules are also uncommon with pulmonary tuberculosis. Fungal infections such as histoplasmosis can cause skin lesions and pulmonary nodules but do not fit well with uveitis.

At this point, "tissue is the issue." A skin nodule would be the easiest site to biopsy. If skin biopsy was not diagnostic, computed tomography (CT) of his chest and abdomen should be performed to identify the next most accessible site for biopsy.

Esophagogastroduodenoscopy (EGD) and colonoscopy showed normal findings, and random biopsies from the stomach and colon were normal. CT of the chest, abdomen, and pelvis performed with the administration of intravenous contrast showed multiple solid opacities in both lung fields up to $1 \mathrm{~cm}$, with enlarged mediastinal and retroperitoneal lymph nodes measuring 1 to $3 \mathrm{~cm}$ in diameter, a left pleural effusion, wall thickening in the right colon, and several nonspecific hypodensities in the liver. A punch biopsy taken from the right chest wall lesion demonstrated chronic inflammation without granulomas. The patient underwent CT-guided biopsy of 1 of the right-sided lung nodules, which revealed noncaseating granulomatous inflammation, fibrosis, and necrosis. Neither biopsy contained malignant cells, and additional stains revealed no bacteria, fungi, or acid fast bacilli.

The retroperitoneal and mediastinal adenopathy are indicative of a widely disseminated inflammatory process. Lymphoma continues to be a concern, though uveitis as an initial presenting problem would very unusual. Although biopsy of the chest wall lesion failed to demonstrate granulomatous inflammation, the most parsimonious explanation is that the skin and lung nodules are both related to a single systemic process.

Granulomas form in an attempt to wall off offending agents, whether foreign antigens (talc, certain medications), infectious agents, or self-antigens. Review of histopathology and microbiologic studies are useful first steps. Stains for bacteria, fungi, or acid-fast organisms may diagnose an infec- 


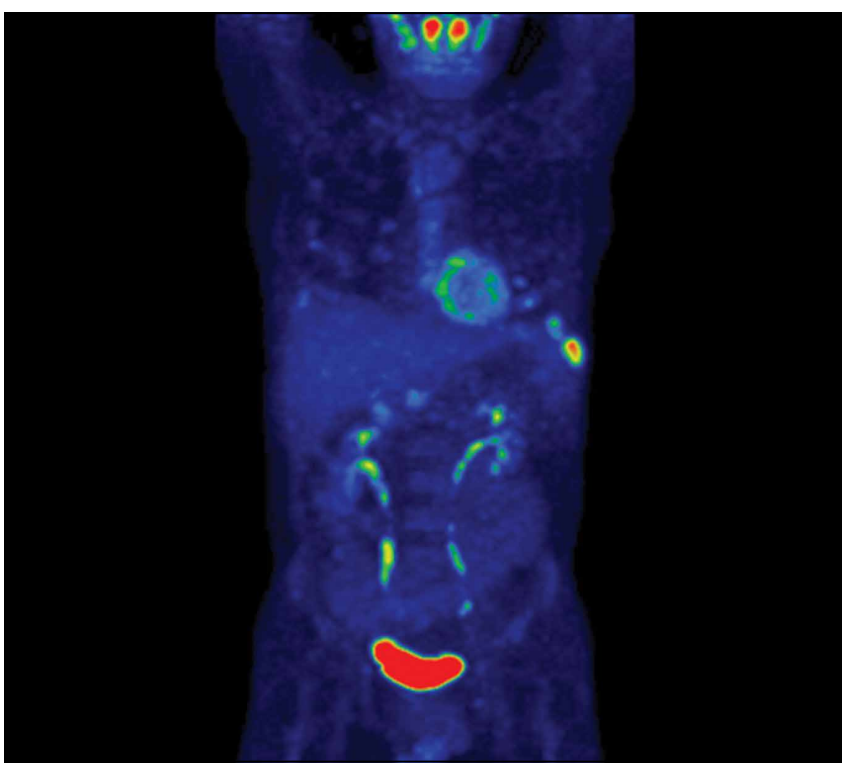

FIG 1. Three dimensional maximum intensity projection (MIP) 18F FDG PET demonstrating tracer activity in lymph nodes in the neck, and several foci in the lungs and costae. Physiological activity in the heart, kidneys, and bladder.

tious cause, such as tuberculosis, leprosy, syphilis, fungi, or cat scratch disease. Granulomas in association with vascular inflammation would indicate vasculitis. Other autoimmune considerations include sarcoidosis and Crohn disease. Noncaseating granulomas are typically found in sarcoidosis, cat scratch disease, syphilis, leprosy, or Crohn disease, but do not entirely exclude tuberculosis.

The negative infectious studies and lack of classic features of Crohn disease or other autoimmune diseases further point to sarcoidosis as the etiology of this patient's illness. A Norwegian dermatologist first described the pathology of sarcoidosis based upon specimens taken from skin nodules. He thought the lesions were sarcoma and described them as, "multiple benign sarcoid of the skin," which is where the name "sarcoidosis" originated.

Diagnosing sarcoidosis requires excluding other mimickers. Additional testing should include syphilis serologies, rheumatoid factor, and antineutrophilic cytoplasmic antibodies. The latter is associated with granulomatosis with polyangiitis and eosinophilic granulomatosis with polyangiitis, either of which may produce granulomatous inflammation of the lungs, skin, and uvea.

A positron emission tomography (PET)-CT demonstrated in Figure 1 shows bilateral increased fluorodeoxyglucose (FDG) uptake in the lungs, skin, and lymph nodes of the neck, mediastinum, and retroperitoneum, in addition to discrete FDG uptake in the liver. Furthermore, osteolytic changes were noted in several ribs.

At this juncture, PET-CT represents a costly and unnecessary test that does not narrow our diagnostic possibilities sufficiently to justify its use. Osteolytic lesions would be unusu-

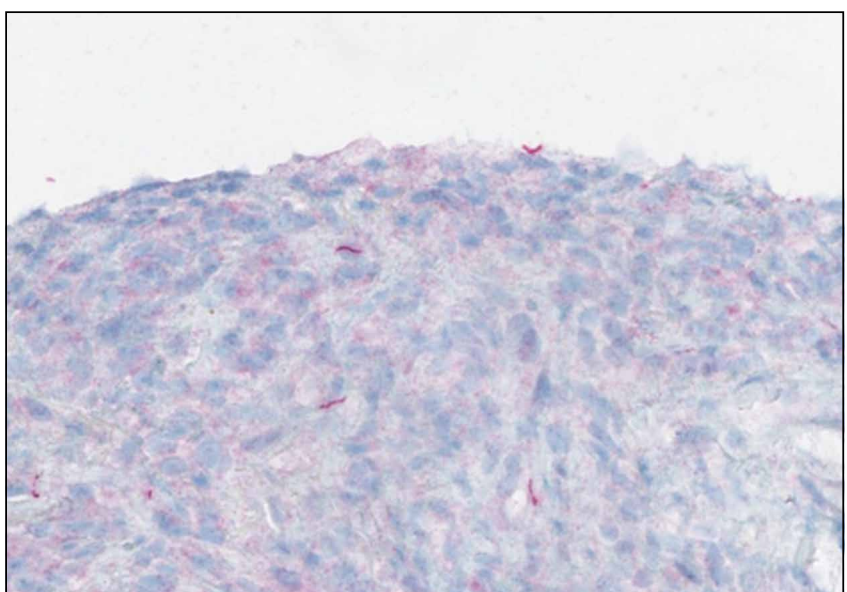

FIG 2. Lung core needle biopsy with spirochetes seen as elongated, delicate, coiling organisms. Immunohistochemical staining (red chromogen) for Treponema pallidum.

al in sarcoidosis and more likely in lymphoma or infectious processes such as tuberculosis. Tests for syphilis and tuberculosis are required, and are a fraction of the cost of a PET-CT.

Another biopsy specimen was taken from a skin lesion on the left chest wall. Pathology revealed granulomatous inflammation again, and additional haematoxylin-eosin staining shown in Figure 2, which had not been performed on the previous specimens, demonstrated spirochetes. Serologies for syphilis were then obtained. Rapid plasma reagin (RPR) titer was elevated at 128 , and IgM and IgG antibody tests to specific Treponema pallidum antigens were also elevated.

With the biopsy revealing spirochetes, and the positive results of a nontreponemal test (RPR) and confirmatory treponemal results, the diagnosis of syphilis is firmly established. Uveitis indicates neurosyphilis and warrants a longer course of intravenous penicillin. Lumbar puncture should be performed.

A lumbar puncture was performed. Cerebrospinal fluid (CSF) contained 9 white blood cells and 73 red blood cells per cubic milliliter; protein concentration was $73 \mathrm{mg} / \mathrm{dL}$, and glucose was $116 \mathrm{mg} / \mathrm{dL}$. Polymerase chain reaction for T. pallidum was negative. Transthoracic ECG and magnetic resonance imaging of the brain were normal. The patient was treated with intravenous penicillin $G$ at 5 million units 4 times daily for 15 days. A PET-CT scan 3 months later revealed complete resolution of the subcutaneous, pulmonary, liver lesions, lymphadenopathy, and uveitis. Repeat treponemal serologies demonstrated a greater than 4-fold decline in titers.

\section{DISCUSSION}

Syphilis is a sexually transmitted disease with increasing incidence worldwide. Untreated infection progresses through 
3 stages. The primary stage is characterized by the appearance of a painless chancre after an incubation period of 2 to 3 weeks. Four to 8 weeks later, the secondary stage emerges as a systemic infection, often heralded by a maculopapular rash with desquamation, frequently involving the soles and palms. Hepatitis, iridocyclitis, and early neurosyphilis may also be seen at this stage. Subsequently, syphilis becomes latent. One-third of patients with untreated latent syphilis will develop tertiary syphilis, typified by late neurosyphilis (tabes dorsalis and general paresis), cardiovascular disease (aortitis), or gummatous disease. ${ }^{1}$

Gummas are destructive granulomatous lesions that typically present indolently, may occur singly or multiply, and may involve almost any organ. It has been suggested that gummas are the immune system's defense to slow the bacteria after attempts to kill it have failed. Histologically, gummas are hyalinized nodules with surrounding granulomatous infiltrate of lymphocytes, plasma cells, and multinucleated giant cells with or without necrosis. In the preantibiotic era, gummas were seen in approximately $15 \%$ of infected patients, with a latency of 1 to 46 years after primary infection. ${ }^{2}$ Penicillin led to a drastic reduction in gummas until the HIV epidemic, which led to the resurgence of gummas at a drastically shortened interval following primary syphilis. ${ }^{3}$

Most commonly, gummas affect the skin and bones. In the skin, lesions may be superficial or deep and may progress into ulcerative nodules. In the bones, destructive gummas have a characteristic "moth-eaten" appearance. Less common sequelae of gummas incude gummatous hepatitis, perforated nasal septum (saddle nose deformity), or hard palate erosions. ${ }^{2,4}$ Rarely, syphilis involves the lungs, appearing as nodules, infiltrates, or pleural effusion. ${ }^{5}$

Ocular manifestations occur in approximately $5 \%$ of patients with syphilis, more often in secondary and tertiary stages, and are strongly associated with a spread to the central nervous system. Syphilis may affect any structure of the eye, with anterior uveitis as the most frequent manifestation. Partial or complete vision loss is identified in approximately half of the patients with ocular syphilis and may be completely reversed by appropriate treatment. Ophthalmologic findings such as optic neuritis and papilledema imply advanced illness, as do Argyll-Robertson pupils (small pupils that are poorly reactive to light, but with preserved accommodation and convergence). ${ }^{6,7}$ The treatment of ocular syphilis is identical to that of neurosyphilis. The Centers for Disease Control and Prevention recommends CSF analysis in any patient with ocular syphilis. Abnormal results should prompt repeat lumbar puncture every 3 to 6 months following treatment until the CSF results normalize. ${ }^{8}$

The diagnosis of syphilis relies on indirect serologic tests. T. pallidum cannot be cultured in vitro, and techniques to identify spirochetes directly by using darkfield microscopy or DNA amplification via polymerase chain reaction are limited by availability or by poor sensitivity in advanced syphilis. ${ }^{1}$ Imaging modalities including PET cannot reliably differentiate syphilis from other infectious and noninfectious mimickers. ${ }^{9}$ Fortunately, syphilis infection can be diagnosed accurately based on reactive treponemal and nontreponemal serum tests. Nontreponemal tests, such as the RPR and Venereal Disease Research Laboratory, have traditionally been utilized as first-line evaluation, followed by a confirmatory treponemal test. However, nontreponemal tests may be nonreactive in a few settings: very early or very late in infection, and in individuals previously treated for syphilis. Thus, newer "reverse testing" algorithms utilize more sensitive and less expensive treponemal tests as the first test, followed by nontreponemal tests if the initial treponemal test is reactive. ${ }^{8}$ Regardless of the testing sequence, in patients with no prior history of syphilis, reactive results on both treponemal and nontreponemal assays firmly establish a diagnosis of syphilis, obviating the need for more invasive and costly testing.

In patients with unexplained systemic illness, clinicians should have a low threshold to test for syphilis. Testing should be extended to certain asymptomatic individuals at higher risk of infection, including men who have sex with men, sexual partners of patients infected with syphilis, individuals with HIV or sexually-transmitted diseases, and others with high-risk sexual behavior or a history of sexually-transmitted diseases. ${ }^{8}$ As the discussant points out, earlier consideration of and testing for syphilis would have spared the patient from unnecessary and costly EGD, colonoscopy, PET-CT scanning, and 3 biopsies.

Syphilis has been known to be a horribly destructive disease for centuries, earning the moniker "morbo serpentino" (serpentine disease) from the Spanish physician Ruiz Diaz de Isla in the 1500 s. $^{10}$ In the modern era, physicians must remember to consider the diagnosis of syphilis in order to effectively mitigate the harm from this resurgent disease when it attacks our patients.

\section{TEACHING POINTS}

- Syphilis, the great imposter, is rising in incidence and should be on the differential diagnosis in all patients with unexplained multisystem inflammatory disease.

- A cost-effective diagnostic approach to syphilis entails serologic testing with treponemal and nontreponemal assays.

- Unexplained granulomas, especially in the skin, bone, or liver, should prompt consideration of gummatous syphilis.

- Ocular syphilis may involve any part of the visual tract and is treated the same as neurosyphilis.

Disclosure: Dr. Weinreich has received payment for lectures from Boehringer er Ingelheim, Astra Zeneca, TEVA and Novartis in 2016. All other contributors have nothing to report.

\section{References}

1. French P. Syphilis. BMJ. 2007;334:143-147

2. Singh AE, Romanowski B. Syphilis: Review with emphasis on clinical, epidemiologic, and some biologic features. Clin Micriobio Rev. 1999;12(2):187-209.

3. Karp G, Schlaeffer F, Jotkowitz A, Riesenberg K. Syphilis and HIV co-infection. Eur J Int Med. 2009; 20:9-13.

4. Pilozzi-Edmonds L, Kong LY, Szabo J, Birnbaum LM. Rapid progression to gumma- 
tous syphilitic hepatitis and neurosyphilis in a patient with newly diagnosed HIV. Int J STD AIDS. 2014;26(13)985-987.

5. David G, Perpoint T, Boibieux A, et al. Secondary pulmonary syphilis: report of a likely case and literature review. Clin Infect Dis. 2006;42(3):e11-e15.

6. Moradi A, Salek S, Daniel E, et al. Clinical features and incidence rates of ocular complications in patients with ocular syphilis. Am J Ophthalmol. 2015;159. 334-343.

7. Aldave AJ, King JA, Cunningham ET Jr. Ocular syphilis. Curr Opin Ophthalmol.
2001;12:433-441.

8. Workowski KA, Bolan GA. Sexually transmitted diseases treatment guidelines, 2015. MMWR Recomm Rep. 2015;64:1-137.

9. Lin M, Darwish B, Chu J. Neurosyphilitic gumma on F18-2-fluoro-2-deoxy-Dglucose (FDG) positron emission tomography: An old disease investigated with new technology. J Clin Neurosc. 2009;16:410-412.

10. de Ricon-Ferraz A. Early work on syphilis: Diaz de Ysla's treatise on the serpentine disease of Hispaniola Island. Int J Dermatol. 1999;38(3):222-227. 